\title{
LINE FORMATION IN INHOMOGENEOUS MAGNETIC FIELDS
}

\author{
R. GOHRING
}

Fraunhofer Institut, Freiburg, Germany

Until now, analytic solutions for the problem of line formation in magnetic fields have been given only under special assumptions concerning either the magnetic field or the atmospheric model. Conditions are given for which the system of differential equations can be solved analytically. For the general case a method is given to solve the system in approximation and analytically. LTE is generally assumed which means that we use the system given by Unno (1956) or that given by Beckers (1969) including the Faraday rotation.

\section{The Separation of the System of Differential Equations}

After transforming the system of differential equations such that the $x$-axis of the coordinate system is in the azimuth direction of the magnetic field for all depths, the matrix $A$ of the system has a very simple form (Göhring, 1970)

$$
\mu \frac{\mathrm{d} \mathbf{I}}{\mathrm{d} \tau}=A \mathbf{I}-\mathbf{S} ; \quad \mathbf{I}=\{I, Q, U, V\} .
$$

The elements of the matrix $A$ are functions of the absorption coefficient $\eta$, the parameter $\varrho$ describing the Faraday-rotation and $\mathrm{d} \phi / \mathrm{d} \tau$, the depth-dependence of the azimuth angle.

It is possible to solve the system analytically if we are able to transform the system (1) into a system with a diagonal matrix. Introducing a matrix $L$ and the transformation

$$
\mathbf{I}=L \mathbf{I}^{\prime}
$$

we get

$$
\mu \frac{\mathrm{d} \mathbf{I}^{\prime}}{\mathrm{d} \tau}=\left\{L^{-1} A L-\mu L^{-1} \frac{\mathrm{d} L}{\mathrm{~d} \tau}\right\} \mathbf{I}^{\prime}-L^{-1} \mathbf{S} .
$$

We have not systematically tried to find a matrix $L$ such, that

$$
D=\left\{L^{-1} A L-\mu L^{-1} \frac{\mathrm{d} L}{\mathrm{~d} \tau}\right\}
$$

is a diagonal matrix - it is possible that there exists none - but we have determined $L$ so that $L^{-1} A L$ is diagonal. It can be shown that this is possible. In this case the columns of $L$ are the normalized eigenvectors of $A$. The components $L_{i j}$ are only functions of the magnetic field strength $H$, the field inclination $\gamma$, the damping constant $a$, the Doppler width $\Delta \lambda_{D}$ and $\mathrm{d} \phi / \mathrm{d} \tau$. 
Now only the first term in $D$ is diagonal and we have to ask for

$$
\mu L^{-1} \frac{\mathrm{d} L}{\mathrm{~d} \tau} \equiv 0
$$

In the general case this term is neither diagonal nor zero. It can be shown that (4) is fulfilled only under the following conditions:

(a) pure longitudinal or transverse field; (b) homogeneous field: $\mathrm{d} H / \mathrm{d} \tau=\mathrm{d} \gamma / \mathrm{d} \tau=$ $\mathrm{d} \phi / \mathrm{d} \tau=0$ and depth independent Voigt-function $\mathrm{d} \alpha / \mathrm{d} \tau=\mathrm{d} / \mathrm{d} \tau\left(\Delta \lambda_{D}\right)=0$.

Assuming (a) Hubenet (1955) has given the general solution of (1), under the assumption (b) the problem was solved by Mattig (1966) and Kjeldseth Moe (1968), both Hubenet and Mattig, and Kjeldseth Moe neglected Faraday rotation. Special solutions with the second assumption are given by Unno (1956), Michard (1961) and Wiehr (1968). If the conditions (a) and (b) are not fulfilled, we have to look whether

or

$$
L^{-1} A L \gg \mu L^{-1} \frac{\mathrm{d} L}{\mathrm{~d} \tau}
$$

$$
L^{-1} A L \approx \mu L^{-1} \frac{\mathrm{d} L}{\mathrm{~d} \tau} .
$$

In the first case we are able to solve the system of differential Equations (1) by the method of the perturbation theory, in the second case we have to integrate the four linear differential equations numerically as Beckers (1969) has done.

\section{The Faraday Rotation as a Perturbation}

It can be shown that it is possible to consider the Faraday rotation as a perturbation. The zero order approximation is then given by

$$
\mu \frac{\mathrm{d} \mathbf{I}^{(0)}}{\mathrm{d} \tau}=\left(1+\eta_{0}\right) \mathbf{I}^{(0)}-\mathbf{S}
$$

the higher approximations can be written in the form

$$
\mu \frac{\mathrm{d} \mathbf{I}^{(i)}}{\mathrm{d} \tau}=\left(1+\eta_{0}\right) \mathbf{I}^{(i)}+\varphi \mathbf{I}^{(i-1)}
$$

$\eta_{0}$ is the absorption matrix, $\varphi$ the matrix of the Faraday rotation. It can be shown that the solutions of (5) and (6) are members of a convergent series and that, with four iteration steps, the result is better than $1 \%$.

\section{The General Solution of the System}

The possibility to take into account the Faraday rotation as a perturbation and to find a convergent series, the general solution of (1) is reduced to the solution of the zero order approximation (5), the system without Faraday rotation. 
Using the transformation (2) we can write for (5) (with $\left.\mathbf{I}^{(0)}=\mathbf{J}\right)$ :

$$
\mu \frac{\mathrm{d} \mathbf{J}}{\mathrm{d} \tau}=\left\{L^{-1}\left(1+\eta_{0}\right) L-\mu L^{-1} \frac{\mathrm{d} L}{\mathrm{~d} \tau}\right\} \mathbf{J}-L^{-1} S .
$$

Here again $L$ is determined such that $L^{-1}\left(1+\eta_{0}\right) L$ is diagonal (this is possible!) and $\mu L^{-1}(\mathrm{~d} L / \mathrm{d} \tau)$ is assumed to be a small perturbation. All calculations for the atmosphere from Holweger (1967) and Kneer (1970) and with a strong depth-dependence of both the magnetic field strength and the field inclination have shown that this assumption can be made.

With the perturbation theory we can write for the different approximations

$$
\begin{aligned}
& \mu \frac{\mathrm{d} \mathbf{J}^{(0)}}{\mathrm{d} \tau}=L^{-1}\left(1+\eta_{0}\right) L \mathbf{J}^{(0)}-L^{-1} \mathbf{S} \\
& \mu \frac{\mathrm{d} \mathbf{J}^{(i)}}{\mathrm{d} \tau}=L^{-1}\left(1+\eta_{0}\right) L \mathbf{J}^{(i)}-\mu L^{-1} \frac{\mathrm{d} L}{\mathrm{~d} \tau} \mathbf{J}^{(i-1)} .
\end{aligned}
$$

The solution of (7) can be given analytically (the matrix is diagonal!). It has a form like that given by Mattig (1966) and Kjeldseth Moe (1968). The higher approximations (8) can be derived recursively. The series converges relatively fast and the computing time needed is shorter by a factor of 20 using the perturbation theory compared with the pure numerical integration.

\section{References}

Beckers, J. M.: 1969, Solar Phys. 9, 372.

Göhring, R.: 1970, Dissertation, Freiburg.

Holweger, H.: 1967, Z. Astrophys. 65, 365.

Hubenet, H.: 1955, Z. Astrophys. 35, 245.

Kjeldseth Moe, O.: 1968, Solar Phys. 4, 267.

Kneer, F.: 1970, Dissertation, Freiburg.

Mattig, W.: 1966, in Atti del Convegno Sui Campi Magnetici Solari (ed. by M Cimino), G. Barbèra.

Firenze = Mitt. Fraunhofer Inst. Nr. 62.

Michard, R.: 1961, Compt. Rend. 253, 2857.

Unno, W.: 1956, Publ. Astron. Soc. Japan 8, 108.

Wiehr, E.: 1968, Dissertation, Göttingen. 